\title{
Pseudo-Differential Operators and Equations in a Discrete Half-Space
}

\author{
Alexander V. Vasilyev ${ }^{a}$, Vladimir B. Vasilyev ${ }^{a}$ \\ ${ }^{a}$ Belgorod National Research University \\ Studencheskaya 14/1, Belgorod 308007, Russia \\ E-mail: alexvassel@gmail.com \\ E-mail(corresp.): vladimir.b.vasilyev@gmail.com \\ Received October 15, 2017; revised June 28, 2018; accepted June 29, 2018
}

\begin{abstract}
We introduce a digital pseudo-differential operator acting in discrete Sobolev-Slobodetskii spaces and consider pseudo-differential equations with such operators in a discrete half-space. The theorem on a general solution of such equations is proved for a special case.
\end{abstract}

Keywords: discrete functional space, digital distribution, digital pseudo-differential operator, discrete pseudo-differential equation, general solution.

AMS Subject Classification: 35S15; 47G30.

\section{Introduction}

A certain theory of pseudo-differential operators and corresponding equations was constructed in the second half of the last century $[3,11,16,17]$, and it includes as usual boundedness theorems in different functional spaces and a certain variant of symbolic calculus. But for discrete situation there is no any variant of such a theory although there are a lot of approximate constructions for solving simplest kinds of pseudo-differential equations, for example singular integral and similar equations $[1,6,7,8,9,10,13,15,18]$. Moreover, there are some recent studies for these discrete situations from algebraic or symbolic calculus point of view on the whole $m$-dimensional lattice $\mathbb{Z}^{m}[2,14]$. But there are principal difficulties to transfer this approach to another discrete domains which are not $\mathbb{Z}^{m}$, for example a discrete half-space or a discrete cone.

We think to exclude this lacuna and to start studying these discrete analogues of pseudo-differential operators and equations. We also believe that such

Copyright (c) 2018 The Author(s). Published by VGTU Press

This is an Open Access article distributed under the terms of the Creative Commons Attribution License (http://creativecommons.org/licenses/by/4.0/), which permits unrestricted use, distribution, and reproduction in any medium, provided the original author and source are credited. 
a discrete theory will help us to justify approximate solving schemes for these equations.

Earlier the authors obtained some initial results for special discrete pseudodifferential operators and equations, namely Calderon-Zygmund operators [19, $22,23]$ including some comparison between discrete and continuous situations [21]. Moreover, we have some initial results for studying pseudo-differential equations and related boundary value problems for discrete domains in $\mathrm{m}$ dimensional space which are different from $\mathbb{Z}^{m}[24,26,27]$. Using the small parameter $h>0$ we hope to obtain existing theory of pseudo-differential operators and boundary value problems on manifolds with a boundary passing to the limit $h \rightarrow 0$, to justify constructing approximate solutions, and to get error estimates between continuous and discrete solutions in appropriate discrete functional spaces.

The main goal of this paper is to prove a theorem on a structure of a general solution for a model discrete elliptic pseudo-differential equation in a discrete half-space.

\section{Discrete Sobolev-Slobodetskii spaces}

\subsection{Discrete Fourier transform}

We will use the following notations. Let $\mathbb{T}^{m}$ be the $m$-dimensional cube $[-\pi, \pi]^{m}, h>0, \hbar=h^{-1}$. We will consider all functions defined on a cube as periodic functions in $\mathbb{R}^{m}$ with the same cube of periods.

If $u_{d}(\tilde{x}), \tilde{x} \in h \mathbb{Z}^{m}$, is a function of a discrete variable, then we call it "discrete function". For such discrete functions one can define the discrete Fourier transform

$$
\left(F_{d} u_{d}\right)(\xi) \equiv \tilde{u}_{d}(\xi)=\sum_{\tilde{x} \in h \mathbb{Z}^{m}} e^{-i \tilde{x} \cdot \xi} u_{d}(\tilde{x}) h^{m}, \quad \xi \in \hbar \mathbb{T}^{m}
$$

if the latter series converges, and the function $\tilde{u}_{d}(\xi)$ is a periodic function on $\mathbb{R}^{m}$ with the basic cube of periods $\hbar \mathbb{T}^{m}$. This discrete Fourier transform preserves basic properties of the integral Fourier transform, particularly the inverse discrete Fourier transform is given by the formula

$$
\left(F_{d}^{-1} \tilde{u}_{d}\right)(\tilde{x})=\frac{1}{(2 \pi)^{m}} \int_{\hbar \mathbb{T}^{m}} e^{i \tilde{x} \cdot \xi} \tilde{u}_{d}(\xi) d \xi, \quad \tilde{x} \in h \mathbb{Z}^{m} .
$$

The discrete Fourier transform is a one-to-one correspondence between the spaces $L_{2}\left(h \mathbb{Z}^{m}\right)$ and $L_{2}\left(\hbar \mathbb{T}^{m}\right)$ with norms

$$
\left\|u_{d}\right\|_{2}=\left(\sum_{\tilde{x} \in h \mathbb{Z}^{m}}\left|u_{d}(\tilde{x})\right|^{2} h^{m}\right)^{1 / 2}, \quad\left\|\tilde{u}_{d}\right\|_{2}=\left(\int_{\xi \in \hbar \mathbb{T}^{m}}\left|\tilde{u}_{d}(\xi)\right|^{2} d \xi\right)^{1 / 2} .
$$

Example 1. Since the definition for Sobolev-Slobodetskii spaces includes partial derivatives, we use their discrete analogue, i.e. divided difference of first order

$$
\left(\Delta_{k}^{(1)} u_{d}\right)(\tilde{x})=h^{-1}\left(u_{d}\left(x_{1}, \ldots, x_{k}+h, \ldots, x_{m}\right)-u_{d}\left(x_{1}, \ldots, x_{k}, \ldots, x_{m}\right)\right),
$$


for which its discrete Fourier transform looks as follows

$$
\left(\widetilde{\Delta_{k}^{(1)} u_{d}}\right)(\xi)=h^{-1}\left(e^{-i h \cdot \xi_{k}}-1\right) \tilde{u}_{d}(\xi) .
$$

Further for the divided difference of second order we have

$$
\begin{aligned}
& \left(\Delta_{k}^{(2)} u_{d}\right)(\tilde{x})=h^{-2}\left(u_{d}\left(x_{1}, \ldots, x_{k}+2 h, \ldots, x_{m}\right)\right. \\
& \left.\quad-2 u_{d}\left(x_{1}, \ldots, x_{k}+h, \ldots, x_{m}\right)+u_{d}\left(x_{1}, \ldots, x_{k}, \ldots, x_{m}\right)\right)
\end{aligned}
$$

and its discrete Fourier transform

$$
\left.\widetilde{\left(\Delta_{k}^{(2)} u_{d}\right.}\right)(\xi)=h^{-2}\left(e^{-i h \cdot \xi_{k}}-1\right)^{2} \tilde{u}_{d}(\xi) .
$$

Thus, for the discrete Laplacian we have

$$
\left(\Delta_{d} u_{d}\right)(\tilde{x})=\sum_{k=1}^{m}\left(\Delta_{k}^{(2)} u_{d}\right)(\tilde{x}),
$$

so that

$$
\left.\widetilde{\left(\Delta_{d} u_{d}\right.}\right)(\xi)=h^{-2} \sum_{k=1}^{m}\left(e^{-i h \cdot \xi_{k}}-1\right)^{2} \tilde{u}_{d}(\xi) .
$$

We will use the discrete Fourier transform to introduce special discrete Sobolev-Slobodetskii spaces which are very convenient for studying discrete pseudo-differential operators and related equations.

\subsection{Definitions and notations}

\subsubsection{Discrete spaces and digital distributions}

Now we will introduce the basic space $S\left(h \mathbb{Z}^{m}\right)$ which consists of discrete functions with finite semi-norms

$$
\left|u_{d}\right|=\sup _{\tilde{x} \in h \mathbb{Z}^{m}}(1+|\tilde{x}|)^{l}\left|\Delta^{(\mathbf{k})} u_{d}(\tilde{x})\right|
$$

for arbitrary $l \in \mathbb{N}, \mathbf{k}=\left(k_{1}, \ldots, k_{m}\right), k_{r} \in \mathbb{N}, r=1, \ldots, m$, where

$$
\Delta^{(\mathbf{k})} u_{d}(\tilde{x})=\Delta_{1}^{k_{1}} \ldots, \Delta_{m}^{k_{m}} u_{d}(\tilde{x})
$$

In other words, the space $S\left(h \mathbb{Z}^{m}\right)$ is a discrete analogue of the Schwartz space $S\left(\mathbb{R}^{m}\right)$ of infinitely differentiable rapidly decreasing at infinity functions. Usually the space of distributions over the basic space $S\left(\mathbb{R}^{m}\right)$ is denoted by $S^{\prime}\left(\mathbb{R}^{m}\right)$.

Digital distribution we call an arbitrary linear continuous functional defined on $S\left(h \mathbb{Z}^{m}\right)$. A set of such digital distributions we will denote by $S^{\prime}\left(h \mathbb{Z}^{m}\right)$, and a value of the functional $f_{d}$ on the basic function $u_{d}$ will be denoted by $\left(f_{d}, u_{d}\right)$.

Together with the space $S\left(h \mathbb{Z}^{m}\right)$ we consider the space $D\left(h \mathbb{Z}^{m}\right)$ consisting of discrete functions with a compact (finite) support. We say that $f_{d}=0$ in the discrete domain $M_{d} \equiv M \cap h \mathbb{Z}^{m}, M \subset \mathbb{R}^{m}$, if $\left(f_{d}, u_{d}\right)=0, \forall u_{d} \in D\left(M_{d}\right)$, where 
$D\left(M_{d}\right) \subset D\left(h \mathbb{Z}^{m}\right)$ consists of discrete functions whose supports belong to $M_{d}$. If we will denote $\widetilde{M}_{d}$ a union of such $M_{d}$, where $f_{d}=0$ then by definition supp $f_{d}=h \mathbb{Z}^{m} \backslash \widetilde{M}_{d}$.

As usual [28] we can define some simplest operations in the space $S^{\prime}\left(h \mathbb{Z}^{m}\right)$ excluding the differentiation (see below), and a convergence is defined as a weak convergence in the space of functionals $S^{\prime}\left(h \mathbb{Z}^{m}\right)$.

If $f_{d}(\tilde{x})$ is a local summable function then one can define the digital distribution $f_{d}$ by the formula

$$
\left(f_{d}, u_{d}\right)=\sum_{\tilde{x} \in h \mathbb{Z}^{m}} f_{d}(\tilde{x}) u_{d}(\tilde{x}) h^{m}, \quad \forall u_{d} \in S\left(h \mathbb{Z}^{m}\right) .
$$

Such distributions we call regular digital distributions. But there are socalled singular digital distributions like the Dirac mass-function $\left(\delta_{d}, u_{d}\right)=$ $u_{d}(0)$, which can not be represented by the above formula (2.1).

\subsubsection{Digital distributions and the Liouville theorem}

Here we will consider our discrete functions from distribution point of view [28]. For simplicity we consider one-dimensional case because a multidimensional situation will be almost the same.

A multiplication by basic function. If $\varphi(\tilde{x})$ is a discrete function such that for some $l$

$$
|\varphi(\tilde{x})| \leq c|\tilde{x}|^{l}, \quad \forall \tilde{x} \in h \mathbb{Z}^{m},
$$

one can define the discrete distribution $\varphi f_{d}$ for arbitrary $f_{d} \in S^{\prime}\left(h \mathbb{Z}^{m}\right)$ by the formula

$$
\left(\varphi f_{d}, u_{d}\right)=\left(f_{d}, \varphi u_{d}\right), \quad \forall u_{d} \in S\left(h \mathbb{Z}^{m}\right) .
$$

$A$ shift. If $f_{d}, u_{d} \in S(h \mathbb{Z})$, we can define the shift $\left(T_{h} f_{d}\right)(\tilde{x}) \equiv f_{d}(\tilde{x}+h)$ by the following formula

$$
\begin{aligned}
\left(T_{h} f_{d}, u_{d}\right) & =\sum_{\tilde{x} \in h \mathbb{Z}^{m}}\left(T_{h} f_{d}\right)(\tilde{x}) u_{d}(\tilde{x}) h=\sum_{\tilde{x} \in h \mathbb{Z}^{m}} f_{d}(\tilde{x}+h) u_{d}(\tilde{x}) h \\
& =\sum_{\tilde{x} \in h \mathbb{Z}^{m}} f_{d}(\tilde{x}) u_{d}(\tilde{x}-h) h=\sum_{\tilde{x} \in h \mathbb{Z}^{m}} f_{d}(\tilde{x})\left(T_{-h} u_{d}\right)(\tilde{x}) h=\left(f_{d}, T_{-h} u_{d}\right),
\end{aligned}
$$

so we can take the following definition for a shift of digital distribution

$$
\left(T_{h} f_{d}, u_{d}\right)=\left(f_{d}, T_{-h} u_{d}\right), \quad \forall u_{d} \in S\left(h \mathbb{Z}^{m}\right) .
$$

A difference operator. For $u_{d} \in S\left(h \mathbb{Z}^{m}\right)$ the difference operators of first order are defined

$$
\left(\Delta_{+}^{(1)} u_{d}\right)(\tilde{x})=\frac{1}{h}\left(u_{d}(\tilde{x}+h)-u_{d}(\tilde{x})\right), \quad\left(\Delta_{-}^{(1)} u_{d}\right)(\tilde{x})=\frac{1}{h}\left(u_{d}(\tilde{x}-h)-u_{d}(\tilde{x})\right),
$$

and thus according to $(2.2)$ we can write for $f_{d} \in S(h \mathbb{Z})$

$$
\left(\Delta_{+}^{(1)} f_{d}, u_{d}\right)=\sum_{\tilde{x} \in h \mathbb{Z}}\left(\Delta^{(1)} f_{d}\right)(\tilde{x}) u_{d}(\tilde{x}) h=\frac{1}{h} \sum_{\tilde{x} \in h \mathbb{Z}} f_{d}(\tilde{x}+h) u_{d}(\tilde{x}) h
$$




$$
\begin{aligned}
& -\frac{1}{h} \sum_{\tilde{x} \in h \mathbb{Z}} f_{d}(\tilde{x}) u_{d}(\tilde{x}) h=\frac{1}{h} \sum_{\tilde{y} \in h \mathbb{Z}} f_{d}(\tilde{y}) u_{d}(\tilde{y}-h) h \\
& -\frac{1}{h} \sum_{\tilde{y} \in h \mathbb{Z}} f_{d}(\tilde{y}) u_{d}(\tilde{y}) h=\sum_{\tilde{y} \in h \mathbb{Z}} f_{d}(\tilde{y})\left(\Delta^{(1)} u_{d}\right)(\tilde{y}) h=\left(f_{d}, \Delta_{-}^{(1)} u_{d}\right) .
\end{aligned}
$$

It implies the following

Definition 1. For digital distribution $f_{d} \in S^{\prime}(h \mathbb{Z})$ the digital distribution $\Delta^{(1)} f_{d}$ is defined by the formula

$$
\left(\Delta_{+}^{(1)} f_{d}, u_{d}\right)=\left(f_{d}, \Delta_{-}^{(1)} u_{d}\right), \quad \forall u_{d} \in S(h \mathbb{Z}) .
$$

Below we will not distinguish $\Delta_{ \pm}$. One can define the divided difference of $k$-th order $\Delta^{(k)} f_{d}$ for a digital distribution $f_{d}$ by induction

$$
\Delta^{(k)} f_{d}=\Delta^{(1)}\left(\Delta^{(k-1)} f_{d}\right) .
$$

We need some difference analogue for a digital distribution supported at the origin. To obtain these properties we need some preliminary results, these are discrete analogues of Schwartz's theorems [28].

Proposition 1. $f_{d} \in S^{\prime}\left(h \mathbb{Z}^{m}\right)$ iff there exist a positive number $C$ and integer $p \geq 0$ such that for arbitrary $u_{d} \in S\left(h \mathbb{Z}^{m}\right)$ the following inequality

$$
\left|\left(f_{d}, u_{d}\right)\right| \leq C\left|u_{d}\right|_{p}
$$

holds, where

$$
\left|u_{d}\right|_{p}=\sup _{k \leq p, \tilde{x} \in h \mathbb{Z}^{m}}(1+|\tilde{x}|)^{p}\left|\left(\Delta^{(k)} u_{d}\right)(\tilde{x})\right| .
$$

Proof. We will prove the necessity only because one can prove the immediately. Let $f_{d} \in S^{\prime}\left(h \mathbb{Z}^{m}\right)$. We will prove this property by contradiction and suppose that there are no such numbers $C$ and $p$. Then there is a sequence $\left\{u_{d, k}\right\}_{k=1}^{\infty}, u_{d, k} \in S\left(h \mathbb{Z}^{m}\right)$, such that

$$
\left|\left(f_{d}, u_{d, k}\right)\right| \geq k\left|u_{d, k}\right|_{k} .
$$

The following sequence

$$
v_{d, k}(\tilde{x})=\frac{u_{d, k}(\tilde{x})}{\sqrt{k}\left|u_{d, k}\right|_{k}}, \quad k=1,2, \ldots
$$

tends to zero in $S\left(h \mathbb{Z}^{m}\right)$ since for $k \geq s, k \geq r$ we have

$$
\left|\tilde{x}^{s} \Delta^{(r)} v_{d, k}(\tilde{x})\right|=\frac{\left|\tilde{x}^{s} \Delta^{(s)} u_{d, k}(\tilde{x})\right|}{\sqrt{k}\left|u_{d, k}\right|_{k}} \leq \frac{1}{\sqrt{k}} .
$$

Since the functional $f_{d}$ is continuous in $S\left(h \mathbb{Z}^{m}\right)$, we obtain

$$
\lim _{k \rightarrow \infty}\left(f_{d}, v_{d, k}\right)=0 .
$$


On the other hand, we obtain from (2.3)

$$
\left|\left(f_{d}, v_{d, k}\right)\right|=\frac{\left|\left(f_{d}, u_{d, k}\right)\right|}{\sqrt{k}\left|u_{d, k}\right|_{k}} \geq \sqrt{k} .
$$

This contradiction proves the Proposition 1.

Lemma 1. If a digital distribution $f_{d} \in S^{\prime}(h \mathbb{Z})$ is supported at zero then it is a finite span of divided differences of $f$ up to $n$-th order. In other words

$$
f_{d}(\tilde{x})=\sum_{k=0}^{n} c_{k}\left(\Delta^{(k)} \delta_{d}\right)(\tilde{x})
$$

Proof. Since supp $f_{d}=\{0\}$, then for arbitrary $k>0$

$$
f_{d}=\varphi(k \tilde{x}) f_{d}
$$

where $\varphi(\tilde{x}) \in S\left(h \mathbb{Z}^{m}\right)$ is equal to 1 in some neighbourhood of 0 , and equals to 0 for $|\tilde{x}|>1$. According to the Proposition 1, we have

$$
\left|\left(f_{d}, u_{d}\right)\right| \leq C\left|u_{d}\right|_{n}, \quad \forall u_{d} \in S\left(h \mathbb{Z}^{m}\right)
$$

for some $C>0, n \geq 0$, non-depending on $u_{d}$.

For arbitrary $u_{d} \in S\left(h \mathbb{Z}^{m}\right)$ we set

$$
u_{d, n}(\tilde{x})=u_{d, n}(\tilde{x})-\sum_{l=0}^{n} \frac{\left(\Delta^{(l)} u_{d}\right)(0)}{l !} \tilde{x}^{l}, \quad v_{k}(\tilde{x})=u_{d, n}(\tilde{x}) \varphi(k \tilde{x}) .
$$

Taking into account that

$$
\begin{aligned}
& \left(\Delta^{(r)} u_{d, n}\right)(\tilde{x})=O\left(|\tilde{x}|^{n+1-r}\right), \quad \tilde{x} \rightarrow \infty(r \leq n), \\
& \left(\Delta^{(s)} \varphi\right)(k \tilde{x})=O\left(k^{s}\right), \quad k \rightarrow \infty,
\end{aligned}
$$

and applying $(2.5)$ to $v_{k}(\tilde{x})$ we obtain

$$
\begin{aligned}
\left|\left(f_{d}, v_{k}\right)\right| \leq C\left|v_{k}\right|_{n} & =C \sup _{l \leq n,|\tilde{x}| \leq \frac{1}{k}}(1+|\tilde{x}|)^{n} \mid \Delta^{(l)}\left(u_{d, n}(\tilde{x}) \varphi(k \tilde{x})\right) \\
& \leq C_{1} \max _{l \leq n,|\tilde{x}| \leq \frac{1}{k}} \sum_{s=0}^{l}\left|\Delta^{(s)} u_{d, n}(\tilde{x})\right|\left|\Delta^{(l-s)} \varphi(k \tilde{x})\right| \\
& \leq C_{2} \max _{l \leq n} \sum_{s=0}^{l} k^{-n-1+s} k^{l-s}=\frac{C_{3}}{k} \rightarrow 0, \quad k \rightarrow \infty .
\end{aligned}
$$

But according to $(2.4)\left(f_{d}, v_{k}\right)$ does not depend on $k$. Thus, we have

$$
\left(f_{d}, v_{1}\right)=\lim _{k \rightarrow \infty}\left(f_{d}, v_{k}\right)=0 .
$$


Therefore, using (2.4) for $k=1$ we obtain the following representation

$$
\begin{aligned}
\left(f_{d}, u_{d}\right) & =\left(\varphi f_{d}, u_{d}\right)=\left(f_{d}, \varphi u_{d}\right)=\left(f_{d}, v_{1}+\sum_{l=0}^{n} \frac{\left(\Delta^{(l)} u_{d}\right)(0)}{l !} \tilde{x}^{l}\right) \\
& =\left(f_{d}, v_{1}\right)+\sum_{l=0}^{n} \frac{\left(\Delta^{(l)} u_{d}\right)(0)}{l !}\left(f_{d}, \tilde{x}^{l} \varphi(\tilde{x})\right)=\sum_{l=0}^{n} C_{l}\left(\Delta^{(l)} \delta_{d}, u_{d}\right)
\end{aligned}
$$

where we set $C_{l}=\left(f_{d}, \tilde{x}^{l} \varphi\right)$. One can easily prove a uniqueness of such representation.

The Fourier transform. Let us note that every digital distribution $f_{d} \in$ $S^{\prime}(h \mathbb{Z})$ can be treated as a distribution $f_{d} \in S^{\prime}(\mathbb{R})$ supported on $h \mathbb{Z}$. Since the Fourier transform for a distribution $f_{d}$ is defined by the standard formula

$$
\left(F f_{d}, u\right)=\left(f_{d}, F u\right), \quad \forall u \in S(\mathbb{R}),
$$

then we have

$$
\left(F \Delta^{(1)} f_{d}, u\right)=\left(f_{d}, \Delta^{(1)} F u\right) .
$$

Now we will calculate the last Fourier transform. For $u \in S(\mathbb{R})$ we have

$$
\left(\Delta^{(1)} \tilde{u}\right)(\xi)=\frac{1}{h}(\tilde{u}(\xi+h)-\tilde{u}(\xi))=\frac{1}{h} \int_{-\infty}^{+\infty}\left(e^{-i h x}-1\right) e^{-i x \xi} u(x) d x,
$$

so that for $f_{d} \in S^{\prime}(\mathbb{R})$

$$
\begin{aligned}
\left(f_{d}, \Delta^{(1)} F u\right) & =\left(f_{d}, F\left(\frac{e^{-i h x}-1}{h} u(x)\right)\right. \\
& =\left(F f_{d}, \frac{e^{-i h x}-1}{h} u\right)=\left(\frac{e^{-i h \xi}-1}{h} F f_{d}, u\right) .
\end{aligned}
$$

If $f_{d} \in S(h \mathbb{Z})$, then

$$
\left(F_{d} \Delta_{+}^{(1)} f_{d}\right)(\xi)=\sum_{\tilde{x} \in h \mathbb{Z}} e^{-i \tilde{x} \cdot \xi} \frac{f_{d}(\tilde{x}+h)-f_{d}(\tilde{x})}{h} h=\frac{e^{-i h \xi}-1}{h}\left(F_{d} f_{d}\right)(\xi),
$$

and the latter formula is agreed with above calculations.

Corollary 1. For the digital distribution

$$
f_{d}(\tilde{x})=\sum_{k=0}^{n} c_{k}\left(\Delta^{(k)} \delta_{d}\right)(\tilde{x})
$$

we have the Fourier transform $\tilde{f}_{d}(\xi)=\sum_{k=0}^{n} c_{k} \zeta^{k}$, where $\zeta=\hbar\left(e^{-i h \xi}-1\right)$.

Remark 1. We use the term "Liouville theorem" because such functions are related with holomorphy properties their Fourier transforms (see, for example, $[3,25])$. 


\subsubsection{Discrete $\mathrm{H}^{\mathrm{s}}$-spaces}

Let us denote $\zeta^{2}=h^{-2} \sum_{k=1}^{m}\left(e^{-i h \cdot \xi_{k}}-1\right)^{2}$ and introduce the following

Definition 2. The space $H^{s}\left(h \mathbb{Z}^{m}\right)$ is a closure of the space $S\left(h \mathbb{Z}^{m}\right)$ with respect to the norm

$$
\left\|u_{d}\right\|_{s}=\left(\int_{\hbar \mathbb{T}^{m}}\left(1+\left|\zeta^{2}\right|\right)^{s}\left|\tilde{u}_{d}(\xi)\right|^{2} d \xi\right)^{1 / 2}
$$

We would like to note that a lot of properties for such spaces were studied in $[4]$.

Further, let $D \subset \mathbb{R}^{m}$ be a domain, and $D_{d}=D \cap h \mathbb{Z}^{m}$ be a discrete domain.

Definition 3. The space $H^{s}\left(D_{d}\right)$ consists of discrete functions from $H^{s}\left(h \mathbb{Z}^{m}\right)$ which supports belong to $\overline{D_{d}}$. A norm in the space $H^{s}\left(D_{d}\right)$ is induced by a norm of the space $H^{s}\left(h \mathbb{Z}^{m}\right)$. The space $H_{0}^{s}\left(D_{d}\right)$ consists of discrete functions $u_{d}$ with a support in $D_{d}$, and these discrete functions should admit a continuation into the whole $H^{s}\left(h \mathbb{Z}^{m}\right)$. A norm in the $H_{0}^{s}\left(D_{d}\right)$ is given by the formula

$$
\left\|u_{d}\right\|_{s}^{+}=\inf || \ell u_{d} \|_{s}
$$

where infimum is taken over all continuations $\ell$.

The Fourier image of the space $H^{s}\left(D_{d}\right)$ will be denoted by $\widetilde{H}^{s}\left(D_{d}\right)$. Such spaces were studied in detail in the paper [4]. Of course, all norms (2.6) are equivalent to the $L_{2}$-norm but this equivalence depends on $h$. Let us note that all constants below in our considerations do not depend on $h$.

\section{Digital pseudo-differential operators and discrete equations}

\subsection{Operators and equations}

Let $\widetilde{A}_{d}(\xi)$ be a periodic function in $\mathbb{R}^{m}$ with the basic cube of periods $\hbar \mathbb{T}^{m}$. Such functions are called symbols. As usual, we will define a digital pseudodifferential operator by its symbol.

Definition 4. A digital pseudo-differential operator $A_{d}$ in a discrete domain $D_{d}$ is called an operator of the following kind

$$
\left(A_{d} u_{d}\right)(\tilde{x})=\sum_{\tilde{y} \in h \mathbb{Z}^{m}} \int_{\hbar \mathbb{T}^{m}} \widetilde{A}_{d}(\xi) e^{i(\tilde{x}-\tilde{y}) \cdot \xi} \tilde{u}_{d}(\xi) d \xi, \quad \tilde{x} \in D_{d} .
$$

An operator $A_{d}$ is called an elliptic operator if

$$
\text { ess } \inf _{\xi \in \hbar \mathbb{T}^{m}}\left|\widetilde{A}_{d}(\xi)\right|>0 .
$$

First, as usual, we define the operator $A_{d}$ on the dense set $S\left(h \mathbb{Z}^{m}\right)$ and then extend it on more general space. 
Remark 2. One can introduce the symbol $\widetilde{A}_{d}(\tilde{x}, \xi)$ depending on a spatial variable $\tilde{x}$ and define a general pseudo-differential operator by the formula

$$
\left(A_{d} u_{d}\right)(\tilde{x})=\sum_{\tilde{y} \in h \mathbb{Z}^{m}} \int_{\hbar \mathbb{T}^{m}} \widetilde{A}_{d}(\tilde{x}, \xi) e^{i(\tilde{x}-\tilde{y}) \cdot \xi} \tilde{u}_{d}(\xi) d \xi, \quad \tilde{x} \in D_{d} .
$$

For studying such operators and related equations one needs to use more fine and complicated technique.

Definition 5. By definition the class $E_{\alpha}$ includes symbols satisfying the following condition

$$
c_{1}\left(1+\left|\zeta^{2}\right|\right)^{\alpha / 2} \leq\left|A_{d}(\xi)\right| \leq c_{2}\left(1+\left|\zeta^{2}\right|\right)^{\alpha / 2}
$$

with universal positive constants $c_{1}, c_{2}$ non-depending on $h$ and the symbol $A_{d}(\xi)$. The number $\alpha \in \mathbb{R}$ is called an order of a digital pseudo-differential operator $A_{d}$.

Obviously, operator $A_{d}$ satisfying (3.1) is an elliptic operator. Using the last definition one can easily get the following property.

Lemma 2. A digital pseudo-differential operator $A_{d} \in E_{\alpha}$ is a linear bounded operator $H^{s}\left(h \mathbb{Z}^{m}\right) \rightarrow H^{s-\alpha}\left(h \mathbb{Z}^{m}\right)$.

We study the equation

$$
\left(A_{d} u_{d}\right)(\tilde{x})=v_{d}(\tilde{x}), \quad \tilde{x} \in D_{d},
$$

assuming that we are interested in a solution $u_{d} \in H^{s}\left(D_{d}\right)$, taking into account $v_{d} \in H_{0}^{s-\alpha}\left(D_{d}\right)$.

Main difficulty for this problem is related to a geometry of the domain $D$. Indeed, if $D=\mathbb{R}^{m}$ then the condition (3.1) guarantees the unique solvability for the equation (3.2). We will consider here only so-called canonical domains and simplest digital pseudo-differential operators with symbols non-depending on a spatial variable $\tilde{x}$. This fact is dictated by using in future the local principle. The last asserts that for a Fredholm solvability of the general equation (3.2) with symbol $A_{d}(\tilde{x}, \xi)$ in an arbitrary discrete domain $D_{d}$, one needs to obtain invertibility conditions for so-called local representatives of the operator $A_{d}$, i.e. for an operator with symbol $A_{d}(\cdot, \xi)$ in a special canonical domain.

Earlier authors have extracted some canonical domains, namely $D=\mathbb{R}^{m}$, $\mathbb{R}_{+}^{m}, C_{+}^{a}$, where $\mathbb{R}_{+}^{m}=\left\{x \in \mathbb{R}^{m}: x=\left(x^{\prime}, x_{m}\right), x_{m}>0\right\}, C_{+}^{a}=\left\{x \in \mathbb{R}^{m}:\right.$ $\left.x_{m}>a\left|x^{\prime}\right|, a>0\right\}$. Methods for studying two last cases are related to special boundary value problems for holomorphic functions [19, 20, 22, 24, 26, 27].

Everywhere below we study the case $D=\mathbb{R}_{+}^{m}$.

\subsection{Periodic Riemann boundary value problem}

For studying the discrete half-space case we need a special technique like continue case $[3,5,12]$. It was found for this case $[20,22]$ the periodic analogue of the Hilbert transform $[3,5,11,12]$ with the parameter $\xi^{\prime}$

$$
\left(H_{\xi^{\prime}}^{p e r} \tilde{u}_{d}\right)\left(\xi^{\prime}, \xi_{m}\right)=\frac{1}{2 \pi i} v \cdot p \cdot \int_{-\hbar \pi}^{\hbar \pi} \cot \frac{h\left(\xi_{m}-\eta_{m}\right)}{2} \tilde{u}_{d}\left(\xi^{\prime}, \eta_{m}\right) d \eta_{m}
$$


where

$$
\begin{aligned}
& v . p . \int_{-\hbar \pi}^{\hbar \pi} \cot \frac{h\left(\xi_{m}-\eta_{m}\right)}{2} \tilde{u}_{d}\left(\xi^{\prime}, \eta_{m}\right) d \eta_{m} \\
& =\lim _{\varepsilon \rightarrow 0+}\left(\int_{-\hbar \pi}^{\xi_{m}-\varepsilon}+\int_{\xi_{m}+\varepsilon}^{\hbar \pi}\right) \cot \frac{h\left(\xi_{m}-\eta_{m}\right)}{2} \tilde{u}_{d}\left(\xi^{\prime}, \eta_{m}\right) d \eta_{m} .
\end{aligned}
$$

This operator generates two projectors

$$
P_{\xi^{\prime}}^{\text {per }}=\frac{1}{2}\left(I+H_{\xi^{\prime}}^{\text {per }}\right), \quad Q_{\xi^{\prime}}^{\text {per }}=\frac{1}{2}\left(I-H_{\xi^{\prime}}^{\text {per }}\right),
$$

which permit to formulate and solve the following problem.

Let us denote $\Pi_{ \pm}$half-strips in the complex plane $\mathbb{C}$

$$
\Pi_{ \pm}=\{z \in \mathbb{C}: z=s+i \tau, s \in[-\pi, \pi], \pm \tau>0\}
$$

and let $H^{ \pm}\left(\hbar \mathbb{T}^{m}\right) \subset L_{2}\left(\hbar \mathbb{T}^{m}\right)$ be subspaces of functions $u\left(\xi^{\prime}, \xi_{m} \pm i \tau\right)$ which admit holomorphic continuation in the strips $\hbar \Pi_{ \pm}$and satisfy the condition

$$
\int_{-\hbar \pi}^{\hbar \pi}\left|u\left(\xi^{\prime}, \xi_{m} \pm i \tau\right)\right|^{2} d \xi_{m}<+\infty, \quad \forall \tau>0, \xi^{\prime} \in \hbar \mathbb{T}^{m-1} .
$$

A statement of the problem: find two functions $\Phi^{ \pm} \in H^{ \pm}\left(\hbar \mathbb{T}^{m}\right)$, which satisfy the linear relation

$$
\Phi^{+}(\xi)=G(\xi) \Phi^{-}(\xi)+g(\xi)
$$

where $G(\xi), g(\xi)$ are given functions defined on $\hbar \mathbb{T}^{m}$.

If $G(\xi) \equiv 1$ then the problem (3.3) is called a jump problem. For $g(\xi) \in$ $L_{2}\left(\hbar \mathbb{T}^{m}\right)$ the jump problem has unique solution $[19,20,22]$

$$
\Phi^{+}=P_{\xi^{\prime}}^{p e r} g, \quad \Phi^{-}=-Q_{\xi^{\prime}}^{p e r} g .
$$

The last assertion correspond to the unique representation as the direct sum

$$
L_{2}\left(\hbar \mathbb{T}^{m}\right)=H^{+}\left(\hbar \mathbb{T}^{m}\right) \oplus H^{-}\left(\hbar \mathbb{T}^{m}\right) .
$$

This fact can be generalized for more wide spaces $H^{s}\left(\hbar \mathbb{T}^{m}\right)$ using a boundedness of the operator $H_{\xi^{\prime}}^{\text {per }}$ in such spaces for small $|s|<1 / 2$ (see also Theorem 1 below).

\section{A general solution}

\subsection{Index of factorization}

To study the general Riemann boundary value problem we will use the following concept. 
Definition 6. Periodic factorization of an elliptic symbol $A_{d}(\xi) \in E_{\alpha}$ is called its representation in the form

$$
A_{d}(\xi)=A_{d,+}(\xi) A_{d,-}(\xi)
$$

where the factors $A_{d, \pm}(\xi)$ admit an analytical continuation into half-strips $\hbar \Pi_{ \pm}$ on the last variable $\xi_{m}$ for almost all fixed $\xi^{\prime} \in \hbar \mathbb{T}^{m-1}$ and satisfy the estimates

$$
\left|A_{d,+}^{ \pm 1}(\xi)\right| \leq c_{1}\left(1+\left|\hat{\zeta}^{2}\right|\right)^{ \pm \frac{\infty}{2}}, \quad\left|A_{d,-}^{ \pm 1}(\xi)\right| \leq c_{2}\left(1+\left|\hat{\zeta}^{2}\right|\right)^{ \pm \frac{\alpha-\infty}{2}}
$$

with constants $c_{1}, c_{2}$ non-depending on $h$,

$$
\hat{\zeta}^{2} \equiv \hbar^{2}\left(\sum_{k=1}^{m-1}\left(e^{-i h \xi_{k}}-1\right)^{2}+\left(e^{-i h\left(\xi_{m}+i \tau\right)}-1\right)^{2}\right), \quad \xi_{m}+i \tau \in \hbar \Pi_{ \pm} .
$$

The number $æ \in \mathbb{R}$ is called an index of periodic factorization.

Remark 3. For an elliptic symbol $A_{d}(\xi)$, such periodic factorization always exists(see $[3,20])$.

For some simple cases one can use the topological formula $[3,20]$

$$
æ=\frac{1}{2 \pi} \int_{-\hbar \pi}^{\hbar \pi} d \arg A_{d}\left(\cdot, \xi_{m}\right),
$$

where $A_{d}\left(\cdot, \xi_{m}\right)$ means that $\xi^{\prime} \in \hbar \mathbb{T}^{m-1}$ is fixed, and the integral is the integral in Stieltjes sense. It means that we need to calculate divided by $2 \pi$ variation of the argument of the symbol $A_{d}(\xi)$ when $\xi_{m}$ varies from $-\hbar \pi$ to $\hbar \pi$ under fixed $\xi^{\prime}$.

Example 2. Let $A_{d}(\xi)=k^{2}+\hat{\xi}^{2}, k \in \mathbb{R}$, such that the condition (3.1) is satisfied, in other words $A_{d}$ is the discrete Laplacian plus $k^{2} I$. The variation of an argument mentioned above can be calculated immediately, and it equals to 1 .

As we will see the index of factorization very influences on the solvability picture of the equation (3.1). For special case we have the following result.

Theorem 1. If the elliptic symbol $\tilde{A}_{d}(\xi) \in E_{\alpha}$ admits periodic factorization with index $æ$ so that $|æ-s|<1 / 2$, then the the equation (3.2) has unique solution in the space $H^{s}\left(D_{d}\right)$ for arbitrary right-hand side $v_{d} \in H^{s-\alpha}\left(D_{d}\right)$,

$$
\tilde{u}_{d}(\xi)=\tilde{A}_{d,+}^{-1}(\xi) P_{\xi^{\prime}}^{p e r}\left(\tilde{A}_{d,-}^{-1}(\xi) \widetilde{\ell v_{d}}(\xi)\right)
$$

Remark 4. It is easy to see that the solution does not depend on choice of continuation $\ell v_{d}$.

Here we consider more complicated case when the condition $|æ-s|<1 / 2$ does not hold. There are two possibilities in this situation, and we consider one case which leads to typical boundary value problems. 
Theorem 2. Let $æ-s=n+\delta, n \in \mathbb{N},|\delta|<1 / 2$. Then a general solution of the equation (3.2) in Fourier images has the following form

$$
\tilde{u}_{d}(\xi)=\tilde{A}_{d,+}^{-1}(\xi) X_{n}(\xi) P_{\xi^{\prime}}^{p e r}\left(X_{n}^{-1}(\xi) \tilde{A}_{d,-}^{-1}(\xi) \widetilde{\ell v_{d}}(\xi)\right)+\tilde{A}_{d,+}^{-1}(\xi) \sum_{k=0}^{n-1} c_{k}\left(\xi^{\prime}\right) \hat{\zeta}_{m}^{k}
$$

where $X_{n}(\xi)$ is an arbitrary polynomial of order $n$ of variables $\hat{\zeta}_{k}=\hbar\left(e^{-i h \xi_{k}}-\right.$ $1), k=1, \ldots, m$, satisfying the condition $(3.1), c_{k}\left(\xi^{\prime}\right), j=0,1, \ldots, n-1$, are arbitrary functions from $H^{s_{k}}\left(h \mathbb{T}^{m-1}\right), s_{k}=s-\mathfrak{Q}+k-1 / 2$.

The a priori estimate

$$
\left\|u_{d}\right\|_{s} \leq a\left(\|f\|_{s-\alpha}^{+}+\sum_{k=0}^{n-1}\left[c_{k}\right]_{s_{k}}\right)
$$

holds, where $[\cdot]_{s_{k}}$ denotes a norm in the space $H^{s_{k}}\left(h \mathbb{T}^{m-1}\right)$, and the constant $a$ does not depend on $h$.

Proof. We will use factorization method proving the theorem according to [3], although the same statement can be obtained by the method of periodic Riemann boundary value problem $[19,20,22]$. Since $v_{d} \in H_{0}^{s-\alpha}\left(Q_{d}\right)$, we can continue it to $l v_{d} \in H^{s-\alpha}\left(h \mathbb{Z}^{m}\right)$. Let us introduce

$$
w_{d}(\tilde{x})=l v_{d}(\tilde{x})-\left(A_{d} u_{d}\right)(\tilde{x}),
$$

so that $w_{d}(\tilde{x}) \equiv 0, \forall \tilde{x} \in D_{d}$. Further we write

$$
\left(A_{d} u_{d}\right)(\tilde{x})+w_{d}(\tilde{x})=l v_{d}(\tilde{x})
$$

and apply the discrete Fourier transform

$$
A_{d}(\xi) \tilde{u}_{d}(\xi)+\tilde{w}_{d}(\xi)=\widetilde{l v_{d}}(\xi)
$$

After factorization of our symbol $A_{d}(\xi)$, we have

$$
A_{d,+}(\xi) \tilde{u}_{d}(\xi)+A_{d,-}^{-1}(\xi) \tilde{w}_{d}(\xi)=A_{d,-}^{-1}(\xi) \widetilde{l v_{d}}(\xi)
$$

Now we need to study functional spaces in the last equality. Since $\widetilde{l v_{d}}(\xi) \in$ $\widetilde{H}^{s-\alpha}\left(h \mathbb{Z}^{m}\right)$, then according to properties of $A_{d,-}^{-1}(\xi)$ we obtain $A_{d,-}^{-1}(\xi) \widetilde{l v_{d}}(\xi) \in$ $\widetilde{H}^{s-\infty}\left(h \mathbb{Z}^{m}\right)$. Let $X_{n}(\xi)$ be an arbitrary polynomial of order $n$ of variables $\hat{\zeta}_{k}=\hbar\left(e^{-i h \xi_{k}}-1\right), k=1, \ldots, m$, satisfying the condition (3.1).

Then $X_{n}^{-1}(\xi) A_{d,-}^{-1}(\xi) \widetilde{l v_{d}}(\xi) \in \widetilde{H}^{-\delta}\left(h \mathbb{Z}^{m}\right)$, so that we can write the following decomposition

$$
X_{n}^{-1}(\xi) A_{d,-}^{-1}(\xi) \widetilde{l v_{d}}(\xi)=f_{+}(\xi)+f_{-}(\xi)
$$

where

$$
f_{+}(\xi)=\left(P_{\xi^{\prime}}^{p e r}\left(X_{n}^{-1} A_{d,-}^{-1} \widetilde{l v_{d}}\right)\right)(\xi), \quad f_{-}(\xi)=\left(Q_{\xi^{\prime}}^{p e r}\left(X_{n}^{-1} A_{d,-}^{-1} \widetilde{l v_{d}}\right)\right)(\xi)
$$


according to the jump problem and Theorem 1 . Moreover, $f_{+} \in \widetilde{H}^{-\delta}\left(Q_{d}\right), f_{-} \in$ $\widetilde{H}^{-\delta}\left(h \mathbb{Z}^{m} \backslash Q_{d}\right)$. Therefore,

$$
A_{d,+}(\xi) \tilde{u}_{d}(\xi)+A_{d,-}^{-1}(\xi) \tilde{w}_{d}(\xi)=X_{n}(\xi) f_{+}(\xi)+X_{n}(\xi) f_{-}(\xi)
$$

or in other words,

$$
A_{d,+}(\xi) \tilde{u}_{d}(\xi)-X_{n}(\xi) f_{+}(\xi)=X_{n}(\xi) f_{-}(\xi)-A_{d,-}^{-1}(\xi) \tilde{w}_{d}(\xi) .
$$

Thus, we have that the left-hand side of the last equality belongs to $\widetilde{H}^{s-\infty}\left(Q_{d}\right)$, and the right-hand side belongs to $\widetilde{H}^{s-\infty}\left(h \mathbb{Z}^{m} \backslash Q_{d}\right)$. Now, if we take inverse discrete Fourier transform for both left-hand side and right-hand one we obtain that these are discrete distribution supported on the discrete hyper-plane $h \mathbb{Z}^{m-1}$. Therefore, according to Lemma 1, we obtain

$$
A_{d,+}(\xi) \tilde{u}_{d}(\xi)-X_{n}(\xi) f_{+}(\xi)=\sum_{k=0}^{n} c_{k}\left(\xi^{\prime}\right) \hat{\zeta}_{m}^{k},
$$

or after re-writing

$$
\tilde{u}_{d}(\xi)=\tilde{A}_{d,+}^{-1}(\xi) X_{n}(\xi) P_{\xi^{\prime}}^{p e r}\left(X_{n}^{-1}(\xi) \tilde{A}_{d,-}^{-1}(\xi) \widetilde{\ell v_{d}}(\xi)\right)+\tilde{A}_{d,+}^{-1}(\xi) \sum_{k=0}^{n} c_{k}\left(\xi^{\prime}\right) \hat{\zeta}_{m}^{k} .
$$

The left question is how much summands we need in the right-hand side. Counting principle is a very simple because every summand should belong to the space $\widetilde{H}^{s}\left(\hbar \mathbb{T}^{m}\right)$.

Let us consider the summand $c_{k}\left(\xi^{\prime}\right) \hat{\zeta}_{m}^{k}$. Taking into account that order of $A_{d,+}^{-1}(\xi)$ is $-æ$, we need to verify the finiteness of the $H^{s-\infty}$-norm for $c_{k}\left(\xi^{\prime}\right) \hat{\zeta}_{m}^{k}$. We have

$$
\begin{aligned}
& \left\|c_{k}\left(\Delta_{m}^{(k)} \delta\right)\right\|_{s-æ}^{2}=\left.\int_{\hbar \mathbb{T}^{m}}\left(1+\left|\zeta_{h}^{2}\right|\right)^{s-æ}|| c_{k}\left(\xi^{\prime}\right) \hat{\zeta}_{m}^{k}\right|^{2} d \xi \\
& =\left.\int_{\hbar \mathbb{T}^{m}}\left(1+\left|\zeta_{h}^{2}\right|\right)^{s-æ}|| c_{k}\left(\xi^{\prime}\right)\right|^{2}\left|\hat{\zeta}_{m}^{k}\right|^{2} d \xi \leq a_{1} \hbar^{2(s-æ+k+1 / 2)} \\
& \quad \times \int_{\hbar \mathbb{T}^{m-1}}\left|c_{k}\left(\xi^{\prime}\right)\right|^{2} d \xi^{\prime} \leq a_{2} \int_{\hbar \mathbb{T}^{m-1}}\left(1+\left|\zeta_{h}^{\prime 2}\right|\right)^{s-æ+k+1 / 2}\left|c_{k}\left(\xi^{\prime}\right)\right|^{2} d \xi^{\prime}
\end{aligned}
$$

where $\xi^{\prime}=\left(\xi_{1}, \ldots, \xi_{m-1}\right), \zeta_{h}^{\prime 2}=\hbar^{2} \sum_{k=1}^{m-1}\left(e^{-i h \xi_{k}}-1\right)^{2}$, and the constants $a_{1}, a_{2}$ do not depend on $h$. The last summand should be $(n-1)$-th because for $n$-th summand we obtain a positive growth: for $k=n$ we have $s_{n}=s-æ-n+1 / 2=$ $-n-\delta+n+1 / 2=-\delta+1 / 2>0$.

Corollary 2. Let $æ-s=n+\delta, \in \mathbb{N},|\delta|<1 / 2, v_{d} \equiv 0$. A general solution of the equation (3.2) has the following form

$$
\tilde{u}_{d}\left(\tilde{x}^{\prime}, \tilde{x}_{m}\right)=\tilde{A}_{d,+}^{-1}(\xi) \sum_{k=0}^{n-1} c_{k}\left(\xi^{\prime}\right) \hat{\zeta}_{m}^{k} .
$$

The Theorem 2 implies that if we want to have a unique solution in the case $æ-s=n+\delta, n \in \mathbb{N},|\delta|<1 / 2$, we need some additional conditions to determine uniquely unknown functions $c_{k}\left(\xi^{\prime}\right), k=0,1, \ldots, n-1$. 


\section{Conclusions}

The proof of the Theorem 1 and a solvability theorems for simple boundary value problems for the equation (3.2) will appear in Springer Proc. Math. \& Stat. Consideration and constructions for the left case $æ-s=-n+\delta, n \in$ $\mathbb{N},|\delta|<1 / 2$, will appear in Tatra Mt. Math. Publ.

\section{Acknowledgements}

The authors are very grateful to the referees for critical remarks and suggestions which have helped correcting and improving the paper.

\section{References}

[1] A. Boettcher and B. Silbermann. Analysis of Toeplitz Operators. Springer-Verlag, Berlin, 2006.

[2] L. Botchway, P.G. Kibiti and M. Ruzhansky. Difference equations and pseudodifferential equations on $\mathbf{z}^{n}$. arXiv:1705.07564v1 [math.FA], pp. 1-29, 2017.

[3] G. Eskin. Boundary Value Problems for Elliptic Pseudodifferential Equations. AMS, Providence, 1981.

[4] L. Frank. Spaces of network functions. Math. USSR Sb., 15(2):182-226, 1971. https://doi.org/10.1070/SM1971v015n02ABEH001541.

[5] F.D. Gakhov. Boundary Value Problems. Dover Publications, Mineola, 1981.

[6] I.C. Gohberg and I.A. Feldman. Convolution Equations and Projection Methods for Their Solution. AMS, Providence, 1974.

[7] R. Hagen, S. Roch and B. Silbermann. $C^{*}$-algebras and Numerical Analysis. Marcel Dekker, New York, 2001.

[8] I. Lifanov, L. Poltavskii and G. Vainikko. Hypersingular Integral Equations and their Applications. Chapman \& Hall/CRC, Boca Raton, 2004.

[9] I.K. Lifanov. Singular Integral Equations and Discrete Vortices. VSP, Utrecht, 1996.

[10] I.K. Lifanov and L.N. Poltavskii. Pseudodifference operators and uniform convergence of divided differences. Sbornik: Mathematics, 193(2):205-230, 2002. https://doi.org/10.1070/SM2002v193n02ABEH000627.

[11] S.G. Mikhlin and S. Proessdorf. Singular Integral Operators. Springer-Verlag, Berlin, 1986.

[12] N.I. Muskhelishvili. Singular Integral Equations. North Holland, Amsterdam, 1976.

[13] S. Proessdorf and B. Silbermann. Numerical Analysis for Integral and Related Operator Equations. Birkhauser, Basel, 1991.

[14] V. Rabinovich. Wiener algebra of operators on the lattice $\mu \mathbf{z}^{n}$ depending on the small parameter $\mu>0$. Complex Var. Ell. Equ., 58(6):751-766, 2013. https://doi.org/10.1080/17476933.2011.617042.

[15] J. Saranen and G. Vainikko. Periodic Integral and Pseudodifferential Equations with Numerical Approximation. Springer, Berlin, 2002. https://doi.org/10.1007/978-3-662-04796-5. 
[16] M. Taylor. Pseudodifferential Operators. Princeton University Press, Princeton, 1981.

[17] F. Treves. Introduction to Pseudodifferential Operators and Fourier Integral Operators. Springer, New York, 1980. https://doi.org/10.1007/978-1-4684-87800.

[18] G. Vainikko. Multidimensional Weakly Singular Integral Equations. SpringerVerlag, Berlin - Heidelberg, 1993. https://doi.org/10.1007/BFb0088979.

[19] A. Vasil'ev and V. Vasil'ev. On the solvability of certain discrete equations and related estimates of discrete operators. Doklady Math., 92(2):585-589, 2015. https://doi.org/10.1134/S1064562415050312.

[20] A. V. Vasil'ev and V. B. Vasil'ev. Periodic Riemann problem and discrete convolution equations. Differ. Equ., 51(5):652-660, 2015. https://doi.org/10.1134/S0012266115050080.

[21] A. Vasilyev and V. Vasilyev. Numerical analysis for some singular integral equations. Neural Parallel Sci. Comput., 20(3):313-326, 2012.

[22] A. Vasilyev and V. Vasilyev. Discrete singular operators and equations in a half-space. Azerb. J. Math., 3(1):84-93, 2013.

[23] A.V. Vasilyev and V.B. Vasilyev. Discrete singular integrals in a half-space. In V. Mityushev and M. Ruzhansky(Eds.), Current Trends in Analysis and Its Applications, Trends in Mathematics. Research Perspectives, pp. 663-670, Basel, 2015. Birkhauser. https://doi.org/10.1007/978-3-319-12577-0_72.

[24] V. Vasilyev. Discrete equations and periodic wave factorization. In A. Ashyralyev and A. Lukashov(Eds.), Proceedings of the International Conference on Analysis and Applied Mathematics (ICAAM-2016), volume 1759 of AIP Conf. Proc., p. 020126, Melville, 2016. AIP Publishing. https://doi.org/10.1063/1.4959740.

[25] V. Vasilyev. Discreteness, periodicity, holomorphy, and factorization. In C. Constanda, M. Dalla Riva, P.D. Lamberti and P. Musolino(Eds.), Integral Methods in Science and Engineering, volume 1 of Theoretical Technique, pp. 315-324, New York, 2017. Birkhauser. https://doi.org/10.1007/978-3-319-59384-5_28.

[26] V. Vasilyev. On discrete boundary value problems. In T. Kal'menov and M. Sadybekov(Eds.), Proceedings of the International Conference "Functional Analysis in Interdisciplinary Applications" (FAIA2017), volume 1880 of AIP Conf. Proc., p. 050010, Melville, 2017. AIP Publishing. https://doi.org/10.1063/1.5000647.

[27] V. Vasilyev. The periodic Cauchy kernel, the periodic Bochner kernel, discrete pseudo-differential operators. In T. Simos and C. Tsitouras(Eds.), Proceedings of the International Conference on Numerical Analysis and Applications (ICNAAM-2016), volume 1863 of AIP Conf. Proc., p. 140014, Melville, 2017. AIP Publishing. https://doi.org/10.1063/1.4992321.

[28] V.S. Vladimirov. Generalized Functions in Mathematical Physics. Mir, Moscow, 1979 . 